Proc. Indian Acad. Sci. (Chem. Sci.), Vol. 105, No. 6, December 1993, pp. 677-684.

(C) Printed in India.

\title{
Intramolecular and dissociation dynamics of triatomic molecules: Some results for $\mathrm{HCN}$ and $\mathrm{CO}_{2}$
}

\author{
JAIR BOTINA ${ }^{1}$ and NASEEM RAHMAN ${ }^{2 *}$ \\ ${ }^{2}$ Dipartimento di Scienze Chimiche dell'Università di Trieste, Piazzale Europa 1, 34100 \\ Trieste, Italy \\ ${ }^{2}$ International Institute for Pure and Applied Chemistry, UNIDO, Area di Ricerca, \\ Padriciano 99, 34012 Trieste, Italy
}

\begin{abstract}
Intramolecular dynamics of two linear molecules $\mathrm{HCN}$ and $\mathrm{CO}_{2}$ are studied by computing trajectories, plotting Poincaré sections and calculating Lyapunov exponents. The process of dissociation is then studied by the same technique for $\mathrm{HCN}$ in the presence of a strong $\mathrm{CO}_{2}$ laser. Results of these preliminary calculations unequivocally show a threshold intensity for dissociation which is possible to verify experimentally.
\end{abstract}

Keywords. Intramolecular dynamics; dissociation probability; triatomic molecules; Poincaré sections; Lyapunov exponent.

\section{Introduction}

How much of intramolecular dynamics is understood by performing computations of trajectories integrating the Hamiltonian equation of motion? It turns out that a lot can be learned and the limitations are quite often that of not having enough computer time to carry through all that you would want to do.

In this article, we use some recently utilized and accurate model Hamiltonians for the molecules $\mathrm{CO}_{2}$ and $\mathrm{HCN}$ and examine what classical mechanics can say regarding these molecules. The results are relevant for two cases. In $\S 2$, some results of computation of trajectories in the configuration space of vibrational motion of these molecules are presented utilizing Poincaré sections. The qualitative change in the dynamics on increasing the energy of the molecules is well understood by these computations. The dynamics can thereby be related to the theory of chaos and clear transitions from periodic regular trajectories to non-periodic irregular trajectories are seen. It is difficult to accurately quantify the transition energy by computing the trajectories and examining the Poincare sections. A much better but (computationally) quite expensive method is to calculate the Lyapunov exponents for these trajectories. The change of sign of the Lyapunov exponent with variation of the energy of the system lets one obtain more accurate values of the transition energy. The results of these are discussed in $\S 2$.

The success of the implementation of our own routine for the computation of the trajectories induced us to consider the dynamics of dissociation of these molecules stimulated by an external electromagnetic field. The problem is discussed in $\S 3$ where

* For correspondence 
the first results of these computations are presented. This new technique has now been implemented for the first time to discuss the intramolecular dissociation dynamics as well as to obtain the dissociation probability. It appears to be a rather promising technique especially in view of the near impossibility of obtaining any accurate quantum-mechanical photodissociation probability due to the prohibitive expense of the relevant computation. The threshold field strength of dissociation for HCN has been obtained with an accuracy which should be compared both with theoretical and with eventually experimental methods.

\section{Intramolecular dynamics}

The Hamiltonian model for $\mathrm{CO}_{2}$ molecule is given by:

with

$$
H=H_{1}+H_{2}+H_{12} \text {, }
$$

$$
\begin{aligned}
H_{i} & =\left(P_{i}^{2} / 2 \mu_{i}\right)+V\left(R_{i}\right), \quad i=1,2, \\
H_{12} & =\left(1 / m_{\mathrm{C}}\right) P_{1} P_{2}, \\
V\left(R_{i}\right) & =D\left(1-\exp \left(-\alpha R_{i}\right)\right)^{2},
\end{aligned}
$$

where $D=5.5 \mathrm{ev}$ and $\alpha=3 \cdot 1 \AA^{-1}$.

In the above equation, $R_{i}$ is the displacement of the $\mathrm{CO}$ bond from its equilibrium position, $P_{i}$ is the conjugate momenta corresponding to $R_{i}, 1 / \mu_{i}=1 / m_{\mathrm{C}}+1 / m_{\mathrm{O}}, m_{\mathrm{C}}$ is the mass of the central atom (carbon mass), $m_{\mathrm{o}}$ is the oxygen mass and $i$ refers to the specific bond in the molecule. These bonds are not equivalent if all the three masses are different. $H_{i}$ is the Hamiltonian for the reduced mass $\mu_{i}$ consisting of the kinetic energy $P_{i}^{2} / 2 \mu_{i}$ and the potential $V\left(R_{i}\right)$ which depends only on the position $R_{i}$. The term $H_{12}$ is the coupling between the motion of the two reduced masses (Wilson et al 1955). Since the molecules are linear, we assume that the angle between the two relevant bonds is $180^{\circ}$. Regardless of the choice of the potentials $V\left(R_{i}\right)$, the coupling term $H_{12}$ is exact and plays a crucial role in our consideration. Such a model, while deficient in many respects such as absence of rotation, bending motion, Fermi resonance etc., is still capable of generating a good number of the vibrational levels of the molecule and therefore has been utilized for various studies in the literature (Buch et al 1982; Halonen and Child 1982; Terasaka and Matsushita 1985; Botschwina 1988; Karrlein 1991; Shi and Rabitz 1991).

For HCN, the Hamiltonian (Smith et al 1991) has given the same form as (1) and (2), with

$$
\begin{aligned}
V\left(R_{i}\right) & =V_{i 1} Z_{i}^{2}+V_{i 2} Z_{i}^{3}+V_{i 3} Z_{i}^{4}, \quad i=1,2, \\
Z_{i} & =1-\exp \left(-\alpha_{i} R_{i}\right)
\end{aligned}
$$

and the term $H_{12}$, however, is chosen to be

where

$$
H_{12}=-\left(1 / m_{\mathrm{C}}\right) P_{1} P_{2}+V\left(R_{1}, R_{2}\right)
$$

$$
V\left(R_{1}, R_{2}\right)=V_{1} Z_{1} Z_{2}+V_{2} Z_{1}^{2} Z_{2}+V_{3} Z_{2}^{2} Z_{1}
$$


All the parameters are given in table 1 and the indices 1 and 2 correspond to $\mathrm{CN}$ and $\mathrm{CH}$ coordinates, respectively. These parameters approximately fit the first 40 stretching vibrational levels in the spectrum with an error of about $0.01 \%$ of the energy values and predict the two possible dissociation pathways associated with this molecule within $15 \%$ for the channel $(\mathrm{H}+\mathrm{CN})$ and within $8 \%$ for the channel $(\mathrm{HC}+\mathrm{N})($ Smith et al 1991).

The Hamiltonian equations of motion along with conservation of energy, give rise to a set of three coupled equations for each of these two Hamiltonians. These are then integrated generating the trajectories and the results are plotted as Poincaré sections with the coordinate $R_{2}$ and the momentum $P_{2}$ as the variables. Figures 1 and 2 show a set of these for $\mathrm{CO}_{2}$ and $\mathrm{HCN}$ respectively. These are in ascending order in energy and even a cursory perusal of them shows how the trajectories make the transition from being periodic to chaotic as the energy is increased. A more

Table 1. Parameters for the generalized Morse potential for HCN (Smith et al 1991).

\begin{tabular}{lll}
\hline$\alpha_{\mathrm{CN}}=2.306172 \AA^{-1}$ & $\alpha_{\mathrm{CH}}=1.847393 \AA^{-1}$ & $V_{1}=0.30763 \mathrm{ev}$ \\
$V_{11}=10.99446 \mathrm{ev}$ & $V_{21}=5.69714 \mathrm{ev}$ & $V_{2}=-9.425 \times 10^{-6} \mathrm{ev}$ \\
$V_{12}=0.499332 \mathrm{ev}$ & $V_{22}=0.47528 \mathrm{ev}$ & $V_{3}=3.657 \times 10^{-4} \mathrm{ev}$ \\
$V_{13}=5.954 \times 10^{-3} \mathrm{ev}$ & $V_{23}=8.426 \times 10^{-3} \mathrm{ev}$ & \\
\hline
\end{tabular}
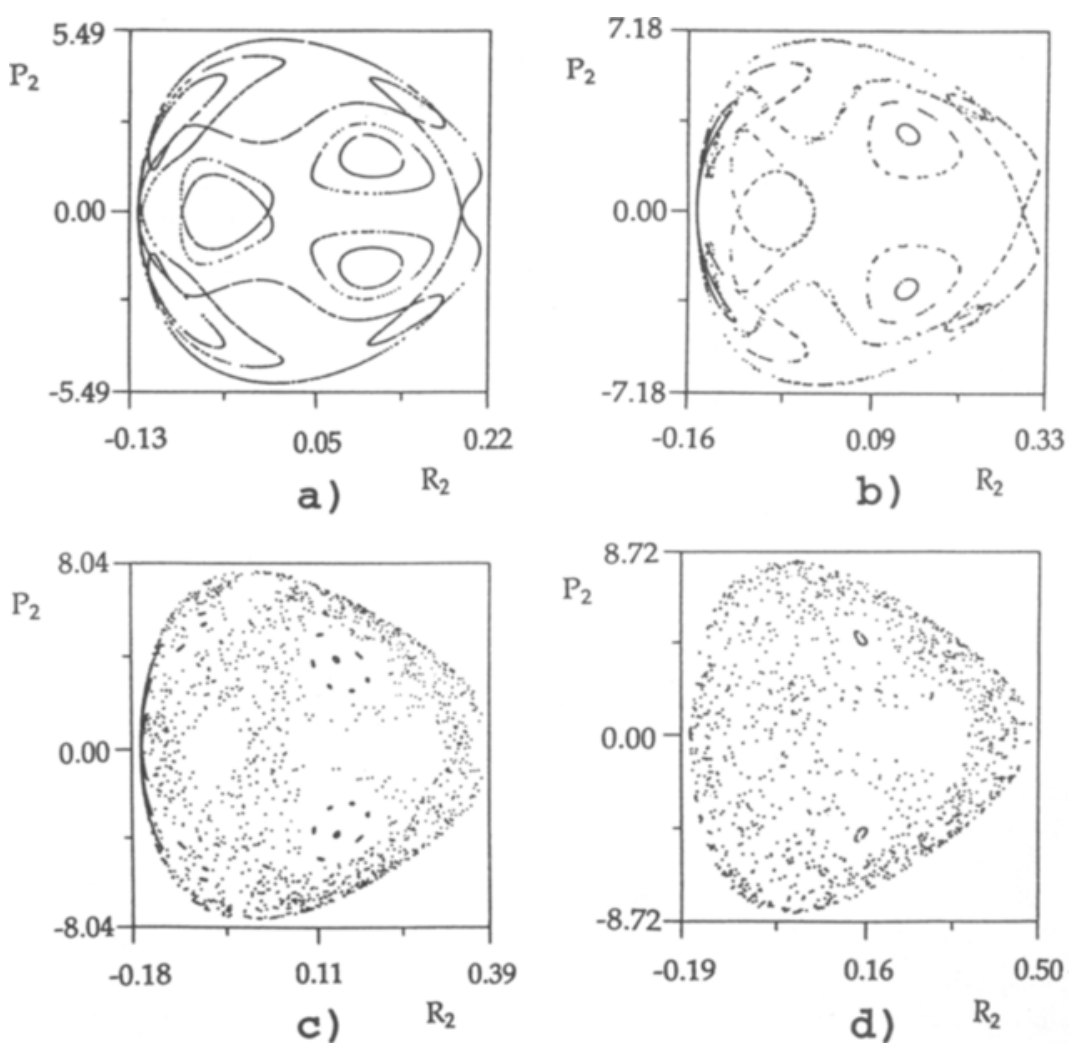

Figure 1. Poincaré section at different values of energy for the $\mathrm{CO}_{2}$ molecule where the two atoms of oxygen are $18 \mathrm{amu}$. (a) $1 \cdot 5$, (b) $2 \cdot 5$, (c) 3.0 and (d) $3 \cdot 5 \mathrm{ev}$. 


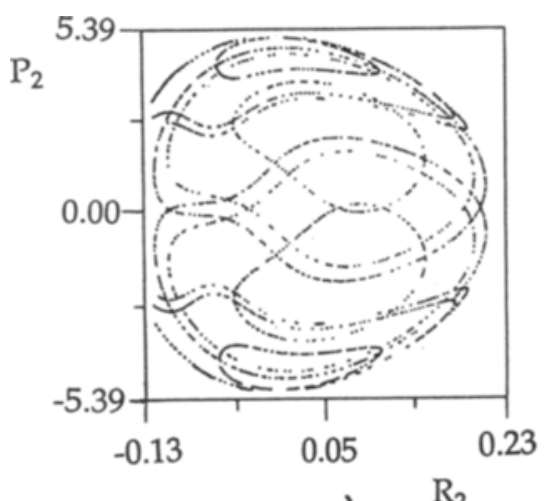

a) $R_{2}$

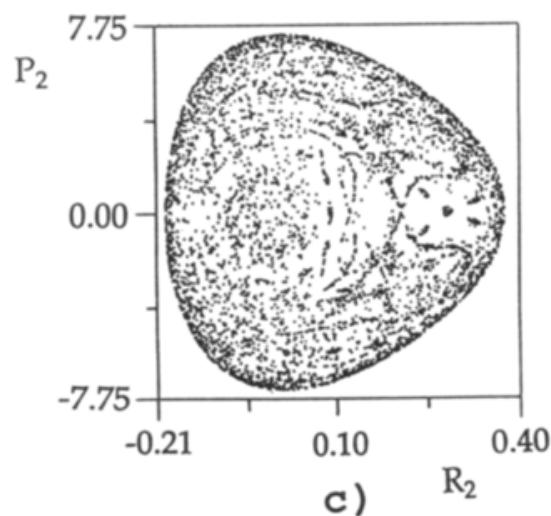

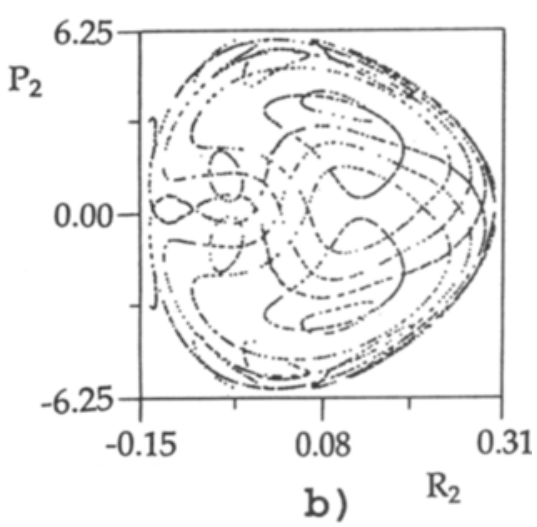

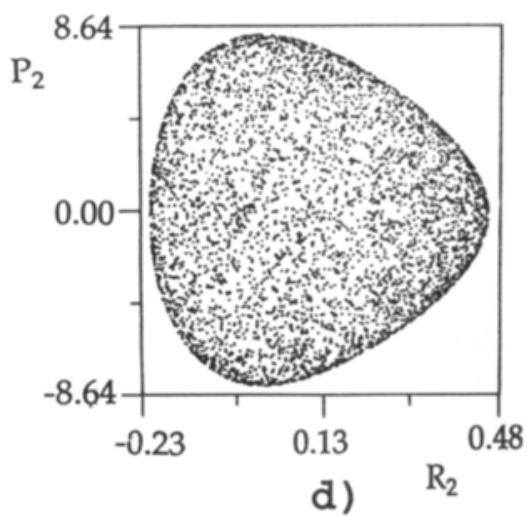

Figure 2. Poincaré section at four values of energy for the molecule $D C^{16} \mathrm{~N}$. (a) 2 , (b) 3 , (c) 4 and (d) 5 ev.

Table 2. Lyapunov exponent at different values of the energy.

\begin{tabular}{lcc}
\hline & $\begin{array}{c}\text { Energy } \\
\text { (ev) }\end{array}$ & $\begin{array}{c}\text { Lyapunov } \\
\text { exponent }\end{array}$ \\
\hline $\mathrm{CO}_{2}$ & 2.50 & -1.0 \\
& 2.76 & -0.2 \\
& 3.00 & 0.5 \\
$\mathrm{HCN}$ & 2.00 & -1.5 \\
& 2.80 & -0.1 \\
& 2.90 & 0.3 \\
\hline
\end{tabular}

quantitative estimate of the energy where such a transition occurs (threshold energy) requires rather extensive calculations involving computation of the Lyapunov exponent (Benettin et al 1976; Shimada and Nagashima 1979; Wolf et al 1985; Meyer 1986). Table 2 indicates where that occurs for ${ }^{12} \mathrm{C}^{16} \mathrm{O}_{2}{ }^{1} \mathrm{H}^{12} \mathrm{C}^{14} \mathrm{~N}$, respectively.

There are two remarks that need to be made and which are generally applicable. First, even the simplest of couplings $-P_{1} P_{2} / m_{\mathrm{C}}$ is able to create the transition to chaos from elementary models of vibrational dynamics. Therefore, we expect, in 
general, to see manifestation of transition to chaos at some energy for all polyatomic molecules in their vibrational motion. Second, chaotic dynamics is strongly and sensitively dependent on the isotopic mass chosen for the molecule (Pichierri 1992; Botina et al 1993).

These computations have served as a starting point for calculation dissociation probability of $\mathrm{HCN}$ by intense laser fields utilizing trajectories and Poincare sections and to investigate the relationship between the threshold energy for chaotic dynamics and the dissociation probability brought about by the external electromagnetic field.

\section{Dissociation dynamies of $\mathrm{HCN}$}

We employed the same type of method described in the previous section to study the laser-induced dissociation of $\mathrm{HCN}$ molecule. The laser field is characterized by the electric field strength and frequency of the laser light. Interaction between the molecule and the laser radiation field is included through the dipole moment function. The Hamiltonian for the laser-molecule interactions is

$$
H_{I}=E_{0} \mu(\mathbf{R}) \cdot \cos (\omega t)
$$

where $E_{0}$ is the electric field strength, $\mu$ is the dipole moment, $t$ is the time, and $\omega$ is the frequency of the laser. The dipole function was taken as

$$
\begin{aligned}
\mu=\left(R_{\mathrm{CH}}, R_{\mathrm{CN}}\right)= & A\left[\left(R_{\mathrm{CN}}+R_{\mathrm{CN}, e q}\right) \exp \left(-\beta R_{\mathrm{CN}}\right)\right. \\
& \left.-\left(R_{\mathrm{CH}}+R_{\mathrm{CH}, e q}\right) \exp \left(-\beta R_{\mathrm{CH}}\right)\right],
\end{aligned}
$$

where $A$ is a constant proportional to the permanent dipole of the molecule in its

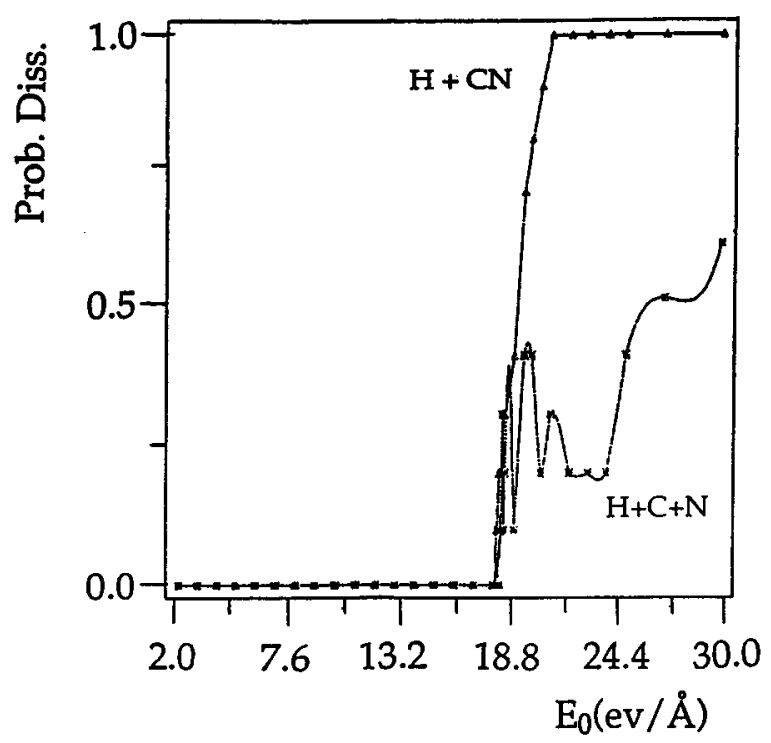

Figure 3. Classical probability of dissociation in function of the electric field strength (ev/ $\AA$ ) where the frequency of the laser is $0.114 \mathrm{ev}$. The number of initial conditions is 20 . 

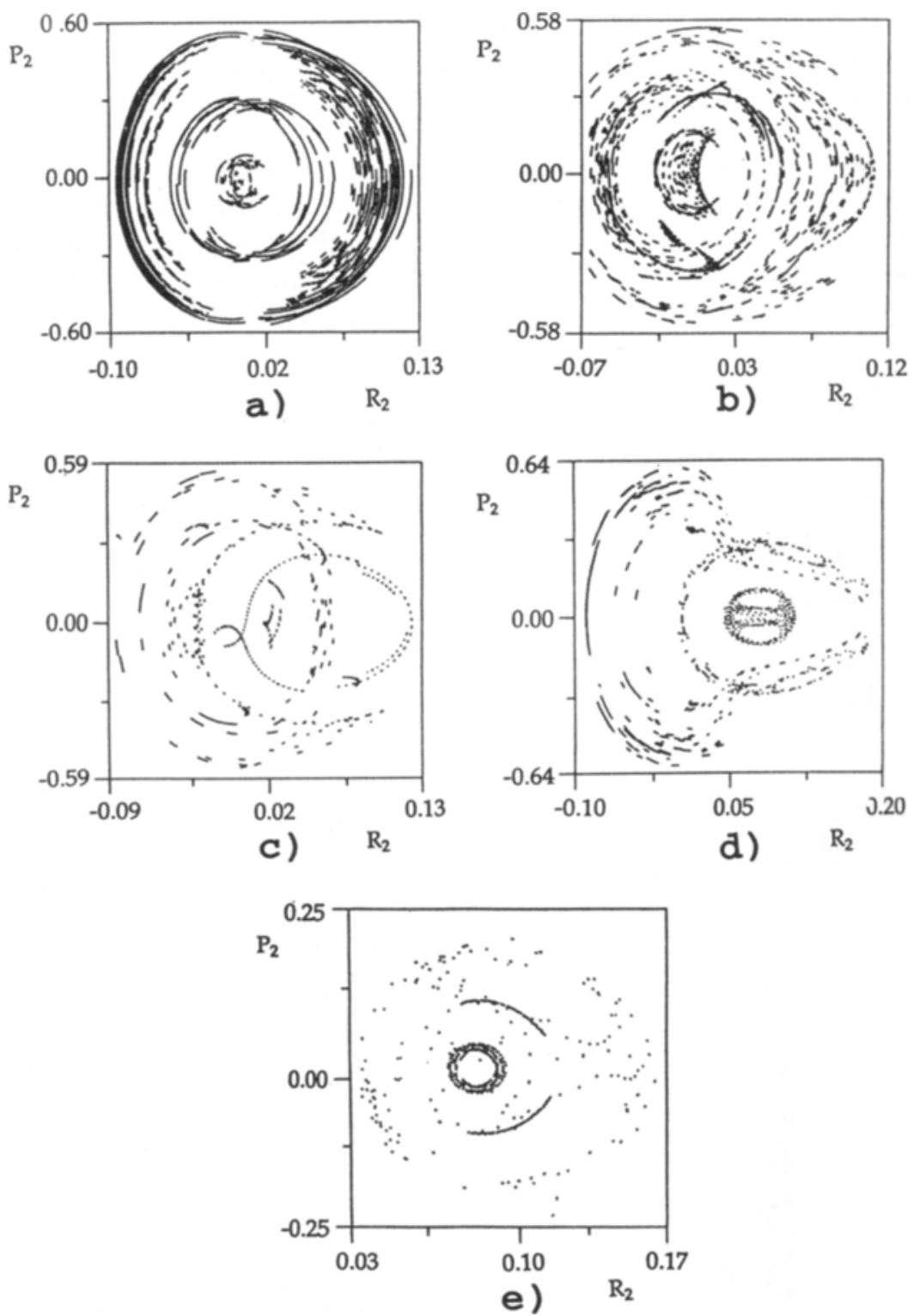

Figure 4. Poincaré section at different values of the electric field strength where the frequency of laser is $0 \cdot 114 \mathrm{ev}$ for the molecule of $\mathrm{HCN}$. (a) 5 , (b) 10 , (c) 15 , (d) 18 and (e) $19 \mathrm{ev} / \AA$.

equilibrium, $R_{i}$ is the displacement from the equilibrium position, $R_{i, e q}$, of each bond $i$, and $\beta$ is a constant that is taken equal to one. We expect this dipole function to be a good approximation because the force constants of $\mathrm{C}-\mathrm{H}$ and $\mathrm{C}-\mathrm{N}$ are not very different from the corresponding force constants in HCN (Smith et al 1987; Botschwina 1988).

In order to study the laser-induced dissociation probability of the HCN molecule, it is necessary to calculate the classical probability of dissociation starting from a set 
Table 3. Lyapunov exponent and the maximum value of energy reached at different values of the electric field strength where the frequency of laser is $0.114 \mathrm{ev}$ for the molecule of $\mathrm{HCN}$. At $19 \mathrm{ev} / \AA$ there is dissociation.

\begin{tabular}{|c|c|}
\hline $\begin{array}{l}\text { Electromagnetic } \\
\text { strength } \\
\text { (ev/A) }\end{array}$ & $\begin{array}{l}\text { Lyapunov } \\
\text { exponent }\end{array}$ \\
\hline 5 & 0.0050 \\
\hline 10 & $0-0224$ \\
\hline 15 & 0.0327 \\
\hline 18 & $0-0500$ \\
\hline 19 & 0.2500 \\
\hline
\end{tabular}

of initial conditions at a specific energy. The probability is given by

$$
P_{\mathrm{diss}}(R, T)=\frac{1}{N} \sum_{i=1}^{N} \delta\left(R_{i}(T)\right)
$$

where $N$ is the number of initial conditions, $T$ is the optical cycle, $R$ is the deviation of the relevant bond $(\mathrm{CN}$ or $\mathrm{CH})$ from its equilibrium position and $\delta$ function is defined as

$$
\delta\left(R_{i}\right)=\left(\begin{array}{lll}
1, & \text { if } & R_{i} \geqslant R_{\text {diss }} \\
0, & \text { if } & R_{i}<R_{\text {diss }}
\end{array}\right),
$$

and $R_{\text {diss }}$ is taken equal to $9 \AA$ (at this value the potential energy $=1 \times 10^{-5} \mathrm{ev}$ ). The results are given in figure 3 where we show the classical probability as a function of the field strength $E_{0}(\mathrm{ev} / \AA)$ for the reactions, $\mathrm{HCN} \rightarrow \mathrm{H}+\mathrm{CN}$ and $\mathrm{H}+\mathrm{CN} \rightarrow \mathrm{H}+\mathrm{C}+\mathrm{N}$. It is clear from figure 3 that the threshold field strength for the dissociation of $\mathrm{HCN} \rightarrow \mathrm{H}+\mathrm{CN}$ has a value of $18 \cdot 16 \mathrm{ev} / \AA$.

In figure 4 we show the same Poincaré section (i.e. for $R_{2}$ and $P_{2}$ ) at different values of field strength and a frequency of $0.114 \mathrm{ev}$. These are for field strengths: (a)

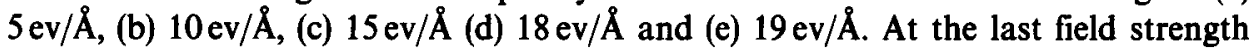
(figure 4e), we have already calculated that the dissociation probability of the molecule is equal to 0.40 for the reaction $\mathrm{HCN} \rightarrow \mathrm{H}+\mathrm{CN}$ and 0.20 for the reaction $\mathrm{H}+\mathrm{CN} \rightarrow$ $\mathrm{H}+\mathrm{C}+\mathrm{N}$ (figure 3). From these figures, we see that at the lowest field strength i.e. at $5 \mathrm{ev} / \AA$, the Poincare section is confined to an almost circular region. With increase of field strength, qualitative changes appear; however, it is difficult to conclude much regarding dissociation from these figures.

Lyapunov exponents at different values of electromagnetic field strength are shown

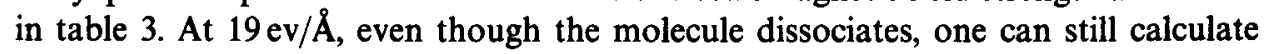
the Lyapunov exponent as long as the calculation is limited to not very long time periods. The values of the Lyapunov exponent jumps from 0.05 at $18 \mathrm{ev} / \AA$ to 0.25 for $19 \mathrm{ev} / \AA$. Therefore one sees that dissociation may be related to deterministic chaos (Smith et al 1987; Botschwina 1988). 


\section{Acknowledgements}

We wish to express our gratitude to the staff of BULL (Area di Ricerca) for a generous grant of computation time on their DPX/2500 computer. Part of the computation and the graphics were performed in the Silicon Graphics workstation provided by IIC. JB wishes to thank ICS for hospitality and financial support during the course of the work.

\section{References}

Benettin G, Galgani L and Strelcyn J M 1976 Phys. Rev. A14 2338

Botina J, Pichierri F and Rahman N 1993 Chem. Phys. Lett. (to be published)

Botschwina P 1988 J. Chem. Soc. Faraday Trans. 841263

Buch V, Gerber R B and Ratner M A 1982 J. Chem. Phys. 765397

Halonen L and Child M S 1982 Mol. Phys. 46239

Karrlein W 1991 J. Chem. Phys. 943293

Meyer H D 1986 J. Chem. Phys. 843147

Pichierri F 1992 Effetti Dinamici sulle Proprietd Statistiche di Stati Vibrazionali Altamente Eccitati in Molecole Triatomiche, thesis, Università degli Studi di Trieste

Shi S and Rabitz H 1991 Comput. Phys. Commun. 6371

Shimada I and Nagashima T 1979 Prog. Theor. Phys. 611605

Smith A M, Jorgensen U G and Lehmann K 1987 J. Chem. Phys. 875649

Smith A M, Klemperer W and Lehmann K 1991 J. Chem. Phys. 945040

Terasaka T and Matsushita T 1985 Phys. Rev. A32 538

Wilson R B Jr, Decius J C and Cross P C 1955 Molecular vibrations. The theory of infrared and Raman vibrational spectra (New York: McGraw-Hill)

Wolf A, Swift J B, Swinney H L and Vastano J A 1985 Physica D16 285 